\title{
Cascade-Reaction-Based Nanodrug for Combined Chemo/Starvation/Chemodynamic Therapy against Multidrug-Resistant Tumors
}

Ying Chen, ${ }^{\dagger}$ Yongchao Yao, ${ }^{\dagger}$ Xueying Zhou, ${ }^{\dagger}$ Chunyan Liao, ${ }^{\dagger}$ Xin Dai, ${ }^{\dagger}$, Jie Liu, $^{\prime \prime}$ Yunlong Yu, ${ }^{\dagger}$ and Shiyong Zhang*,†,†

${ }^{\dagger}$ National Engineering Research Center for Biomaterials and ${ }^{\ddagger}$ College of Chemistry, Sichuan University, 29 Wangjiang Road, Chengdu 610064, China

${ }^{\S}$ Zunyi Medical and Pharmaceutical College, Pingan Road, Xinpu District, Zunyi 56300, China

"State Key Laboratory of Biotherapy and Cancer Center, West China Hospital Sichuan University, Chengdu 610041, China

*E-mail: szhang@scu.edu.cn. Phone/Fax: +86-28-85411109 


\section{Synthesis}

Scheme S1. Synthesis of compound 1
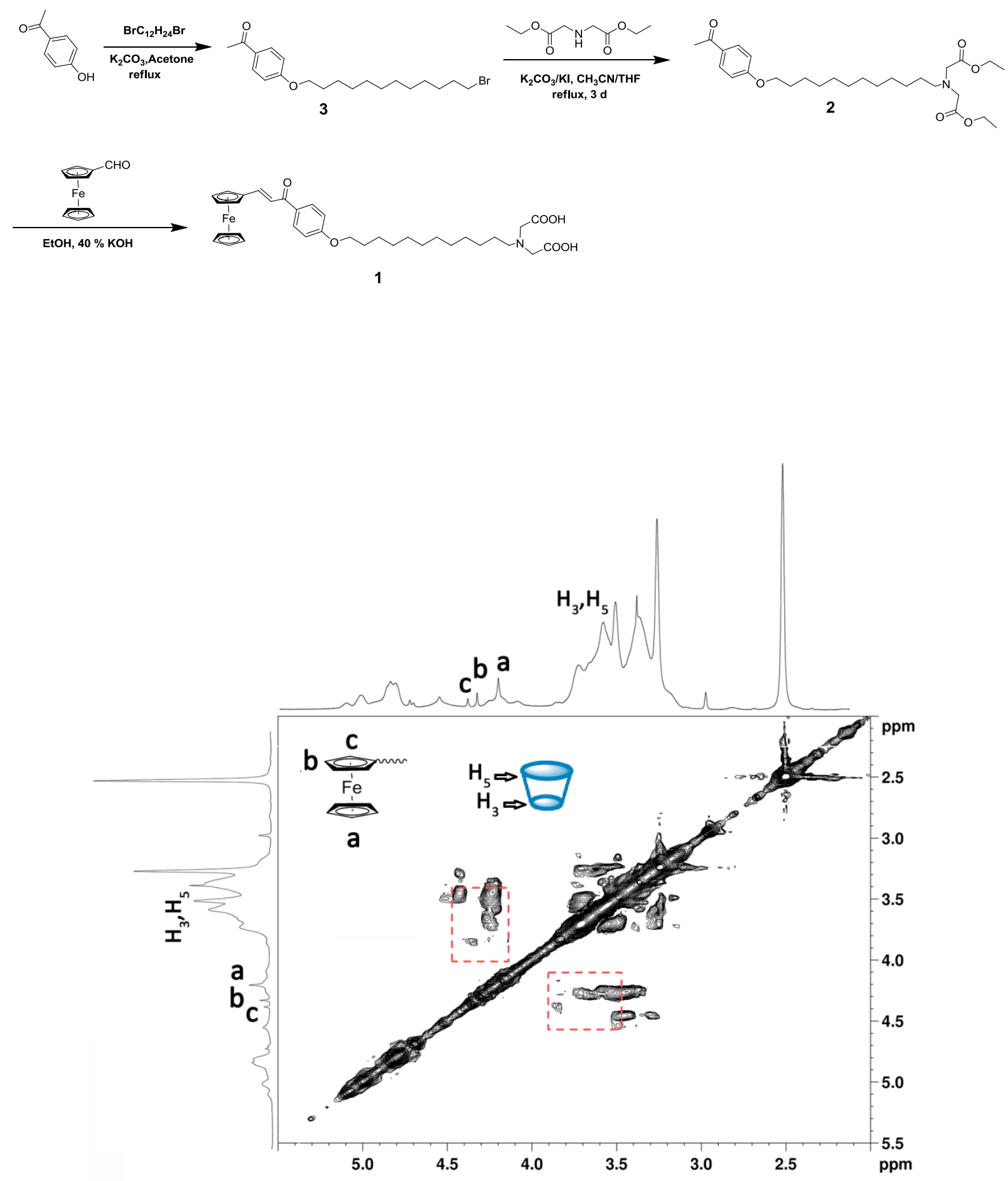

Figure S1. 2D ROESY NMR spectrum of SA in DMSO-d 6 at $25^{\circ} \mathrm{C}$. 


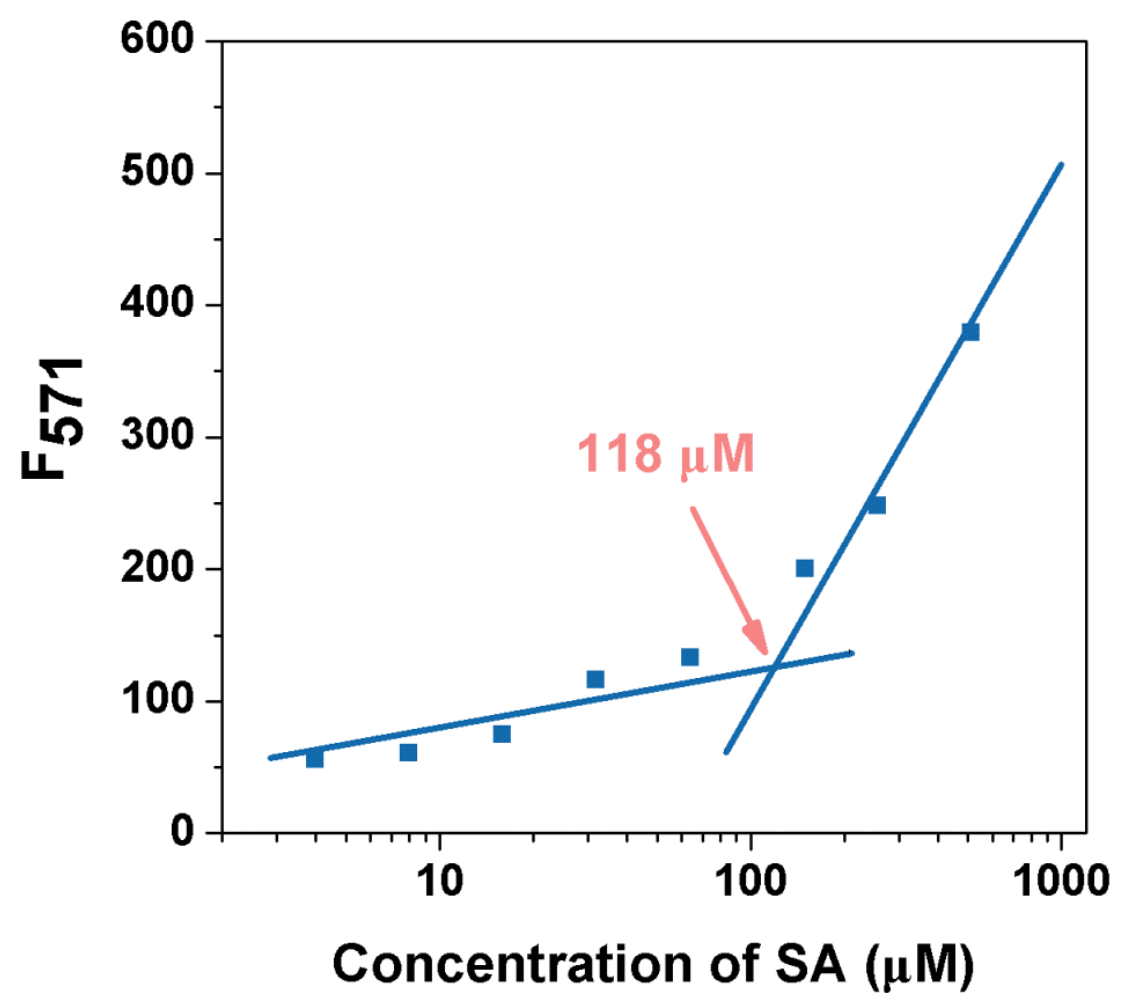

Figure S2. Emission intensity at $571 \mathrm{~nm}\left(F_{571}\right)$ of Nile Red as a function of concentrations of SA in water. $\left[\right.$ Nile Red] $=1.0 \times 10^{-6} \mathrm{~mol} \mathrm{~L}^{-1}, \lambda_{\text {ex }}=485 \mathrm{~nm}$.
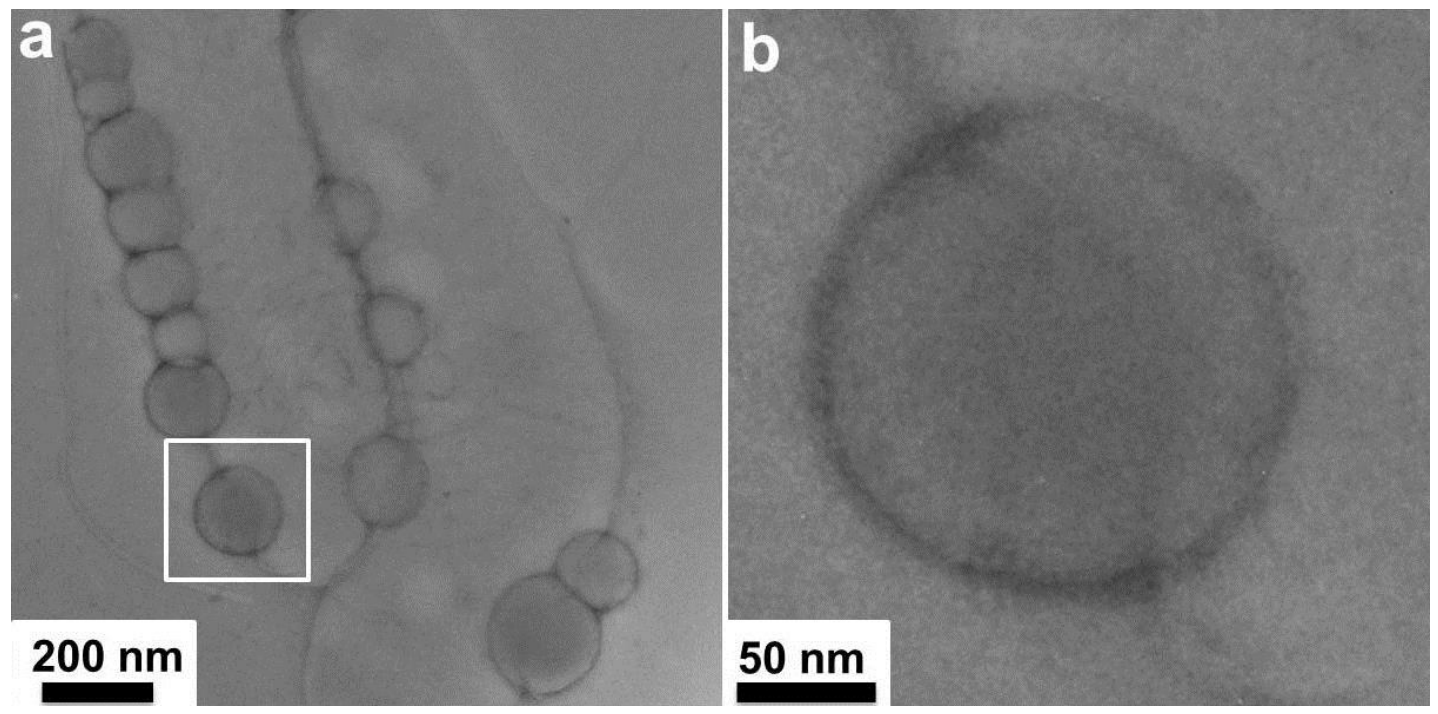

Figure S3. (a) TEM image of SA nanoparticles. (b) A zoomed-in image of the area marked in (a). 


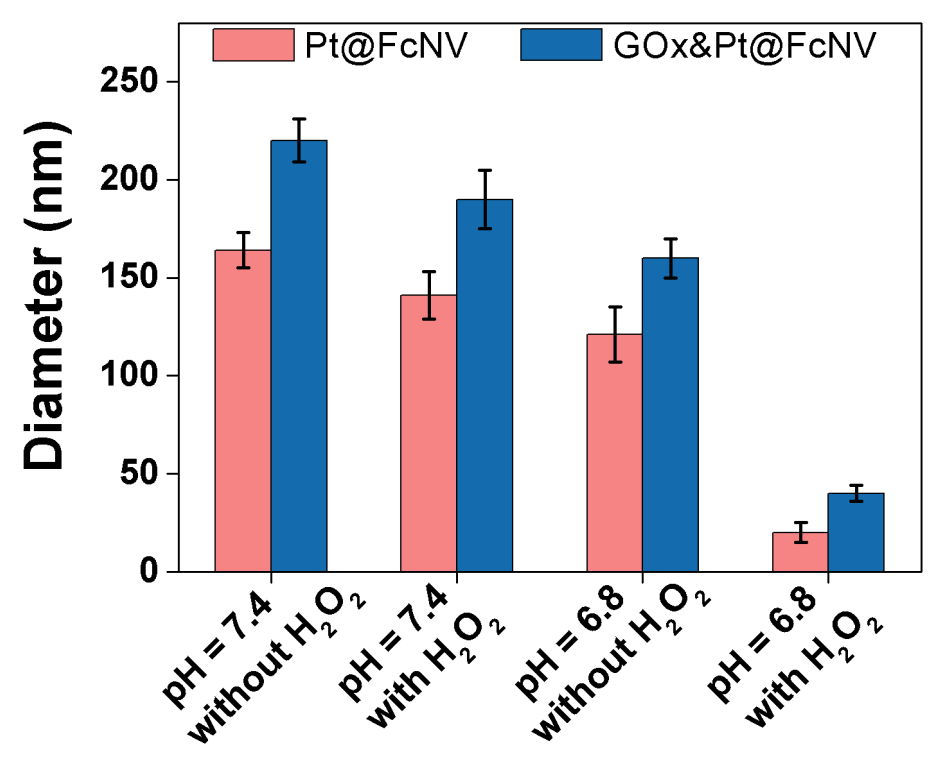

Figure S4. Size changes of Pt@FcNV and GOx\&Pt@FcNV in pH7.4 and pH6.8 PBS buffers with or without $\mathrm{H}_{2} \mathrm{O}_{2}$. Under $\mathrm{pH} 7.4$ without $\mathrm{H}_{2} \mathrm{O}_{2}$, the uniform sizes of $\mathrm{Pt} @ \mathrm{FcNV}$ at 164 nm and GOx\&Pt@FcNV at $220 \mathrm{~nm}$ could keep without obvious changes for several weeks. When $\mathrm{H}_{2} \mathrm{O}_{2}$ was added, the sizes of Pt@FcNV and GOx\&Pt@FcNV could also keep at 141 nm and 190 nm, respectively, suggesting that the addition of $\mathrm{H}_{2} \mathrm{O}_{2}$ didn't destroy the stability of Pt@FcNV and GOx\&Pt@FcNV under the neutral condition. When the $\mathrm{pH}$ was adjusted to 6.8, the sizes of $\mathrm{Pt} @ \mathrm{FcNV}$ and GOx\&Pt@FcNV decreased to 121 nm and 160 nm, respectively, suggesting that Pt@FcNV and GOx\&Pt@FcNV were pH-sensitive. Notably, in the presence of $\mathrm{H}_{2} \mathrm{O}_{2}$ under $\mathrm{pH} 6.8$, the sizes of Pt@FcNV reduced from about 164 to 20 nm, and GOx\&Pt@FcNV reduced from about 220 to 40 nm, indicating that the hydrophobic ferrocene were oxidized into hydrophilic ferrocenium salts, which accelerated the disintegration of Pt@FcNV and GOx\&Pt@FcNV. 

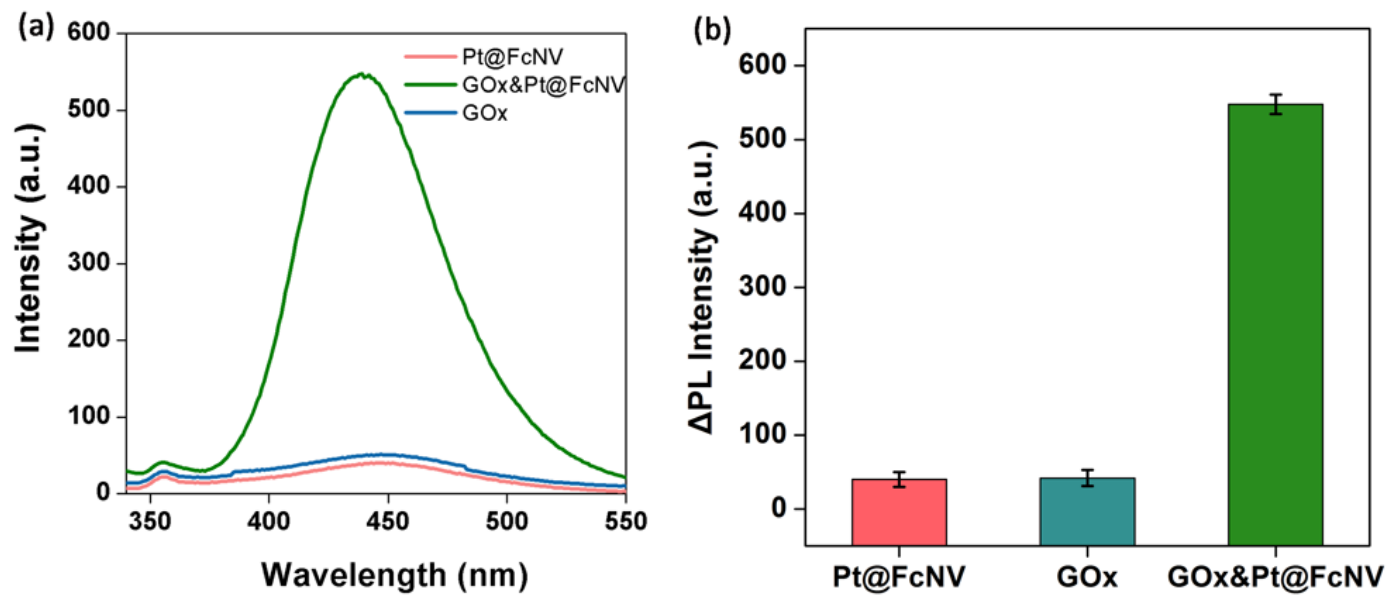

Figure S5. Reaction between hydroxyl radical $\left({ }^{\circ} \mathrm{OH}\right)$ and terephthalic acid (TA). (a) Fluorescence spectra of the PBS solution $(\mathrm{pH}=7.4,1 \mathrm{mM})$ include $\mathrm{Pt} @ \mathrm{FcNV}$ and TA; GOx and TA; GOx\&Pt@FcNV and TA after $12 \mathrm{~h}$ reaction. $\quad\left(\lambda_{\mathrm{ex}}=315 \mathrm{~nm}, \lambda_{\mathrm{em}}=435 \mathrm{~nm}, \mathrm{pH} \sim 7-8 .([\mathrm{SA}]=2.5 \times\right.$ $\left.10^{-3} \mathrm{M},[\mathrm{GOx}]=5.4 \mathrm{U} \mathrm{mL}^{-1},[\mathrm{TA}]=5.0 \times 10^{-4} \mathrm{M}\right)$. (b) Histograms of $\Delta \mathrm{PL}$ intensity showed the catalysis effect of Fc. Error bars were taken from three parallel experiments.

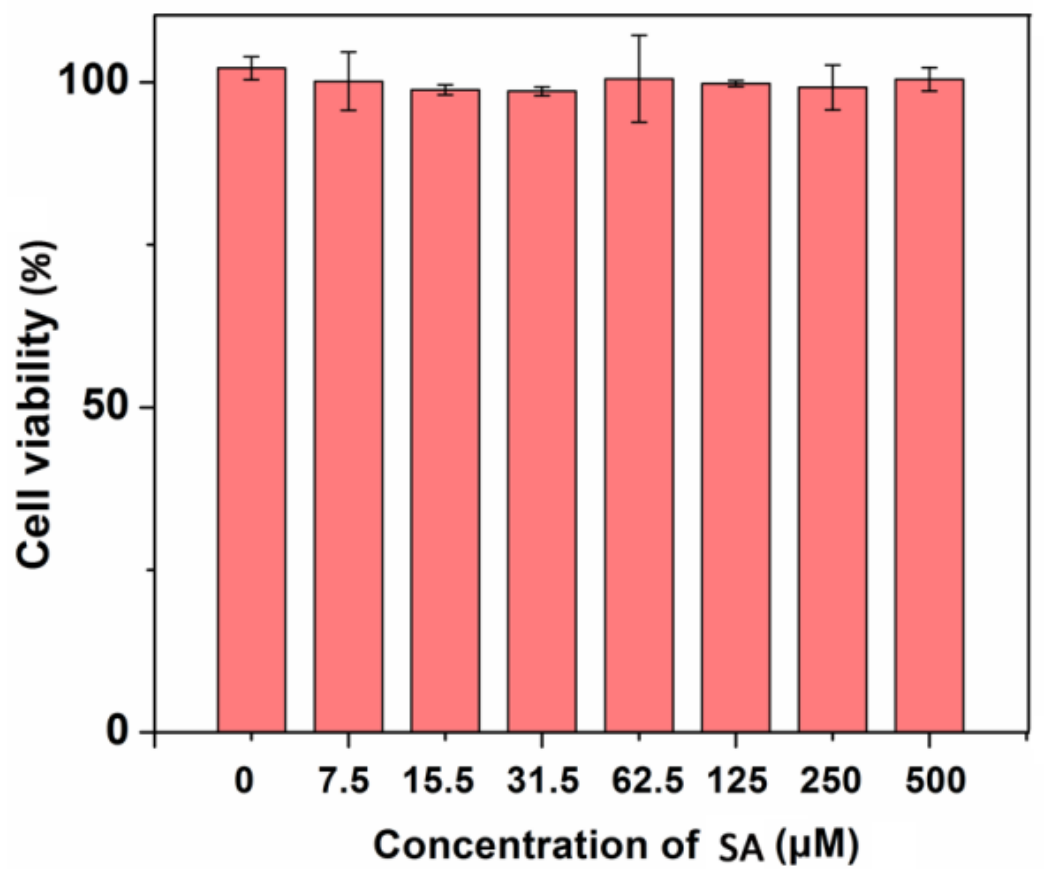

Figure S6. Viability of A549 cells after 48 h of incubation with different concentrations of SA. 


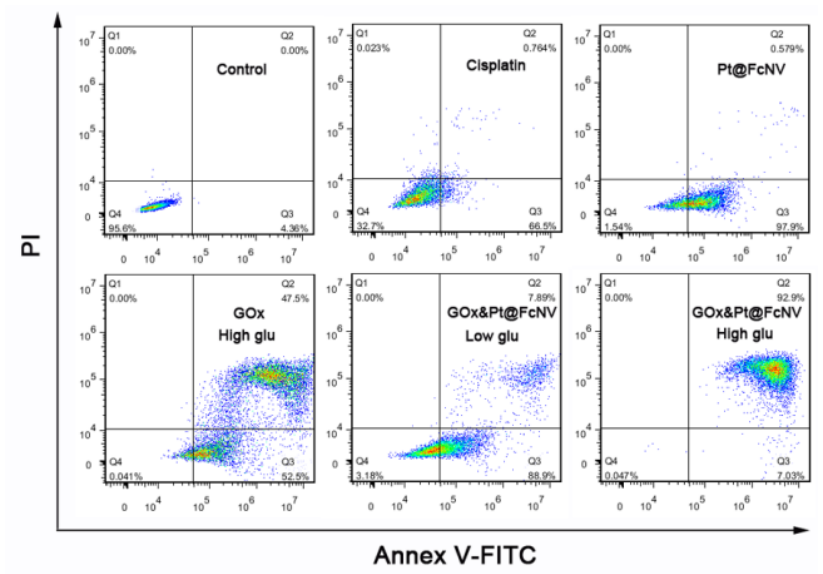

Figure S7. Apoptosis induced by cisplatin, Pt@FcNV, high-glucose GOx, low-glucose GOx\&Pt@FcNV, and high-glucose GOx\&Pt@FcNV against A549/DDP cells for 4 h $\left([\mathrm{Pt}]=25 \mu \mathrm{g} \mathrm{mL}^{-}\right.$ ${ }^{1},[\mathrm{GOx}]=27 \mathrm{mU} \mathrm{mL}^{-1},[\mathrm{SA}]=1.25 \times 10^{-5} \mathrm{M},[$ Low glucose $]=1.0 \mathrm{mg} \mathrm{mL}^{-1}$ glucose, $[$ High glucose $]=$ $4.5 \mathrm{mg} \mathrm{mL}^{-1}$ glucose). Inserted numbers in the profiles present the percentage of the cells in this area. Lower left: living cells; upper left: necrotic cells; lower right: early apoptotic cells; upper right: late apoptotic cells.

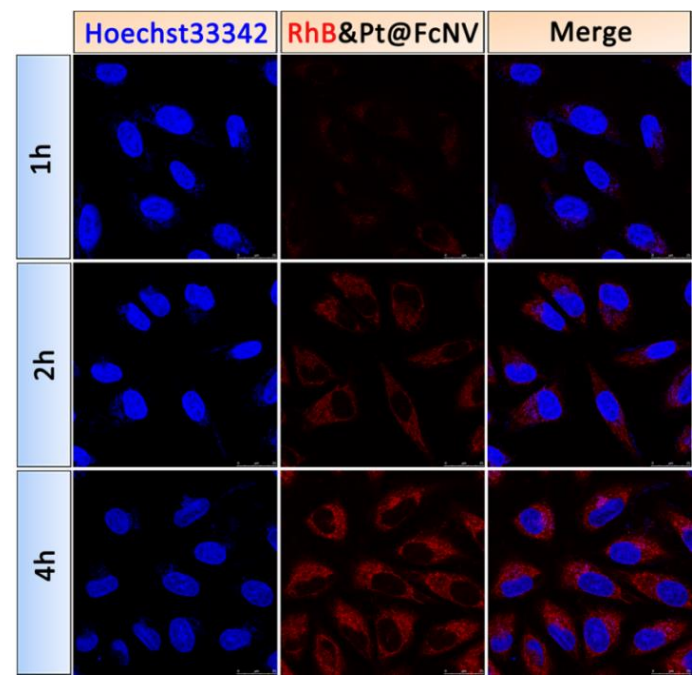

Figure S8. CLSM images of A549/DDP cells incubated with RhB\&Pt@FcNV for 1, 2, and 4 h. For each panel, images from left to right show the cell nuclei stained with Hoechst 33342 (blue), the RhB\&Pt@FcNV in cells (red), and overlays of the two images, respectively. The scale bars are $25 \mu \mathrm{m}$ in all images. 


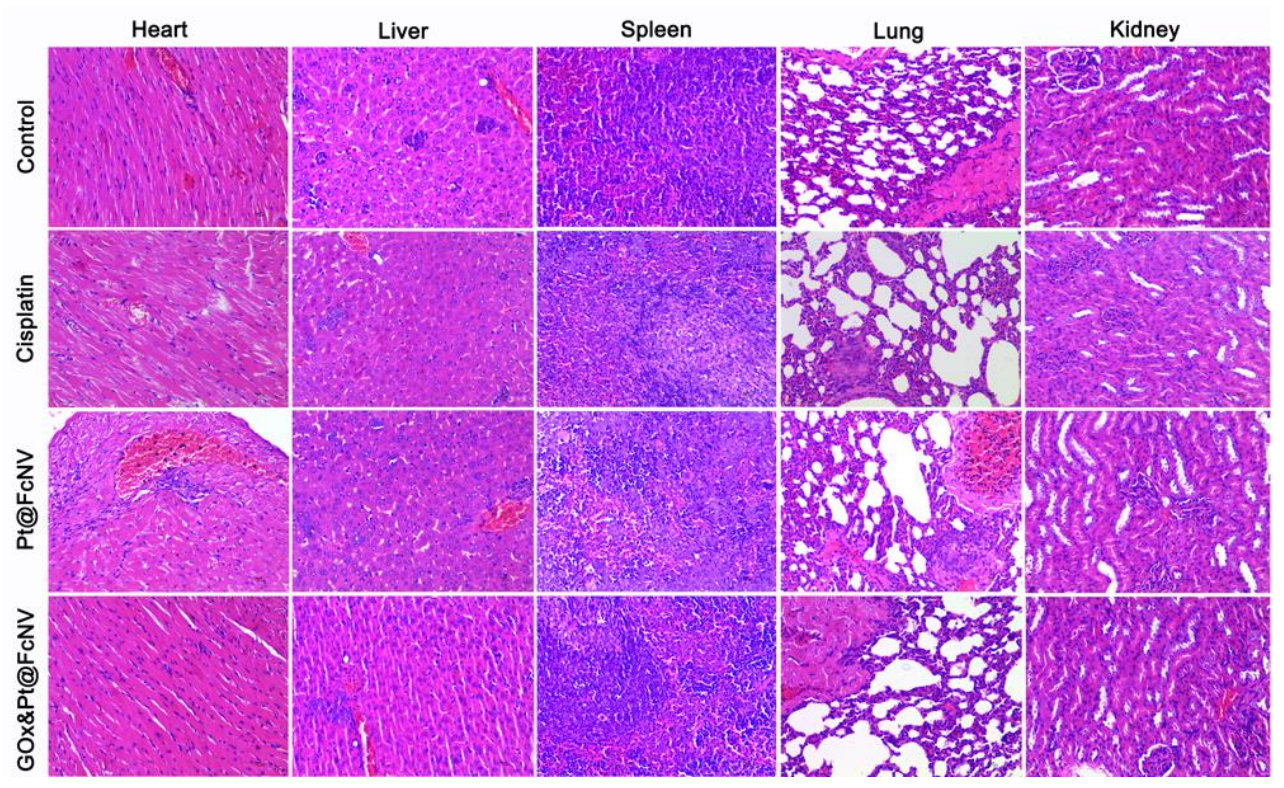

Figure S9. H\&E stained images of major organs including the heart, liver, spleen, lung, and kidney from the treated mice.

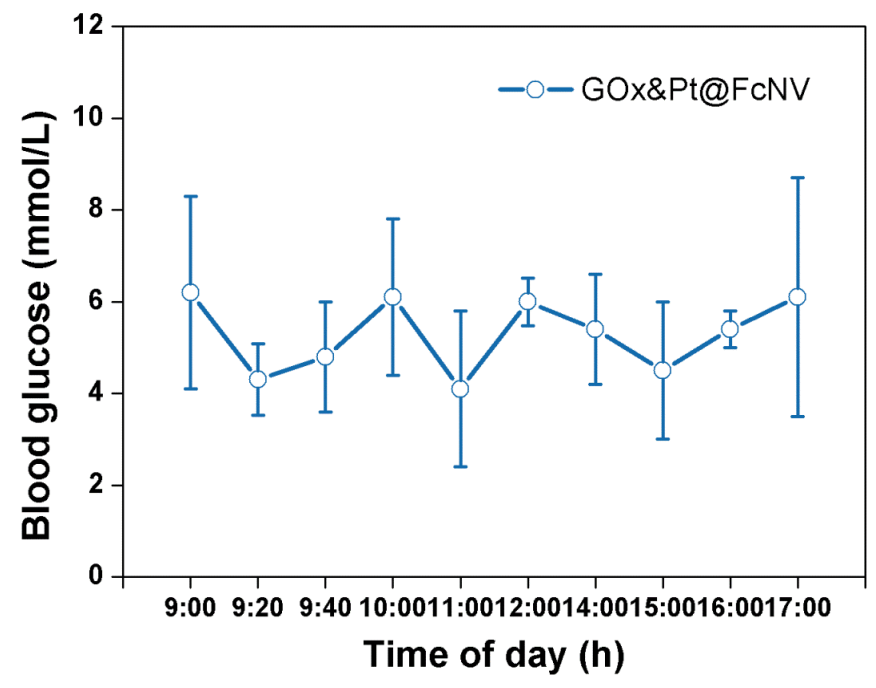

Figure S10. Blood glucose levels of mice in time of day. Mice $(n=3)$ was intravenously injected of GOx\&Pt@FcNV at 09:00 after fasting for 12 hours. Mean values and error bars are defined as mean and standard deviation, respectively. 

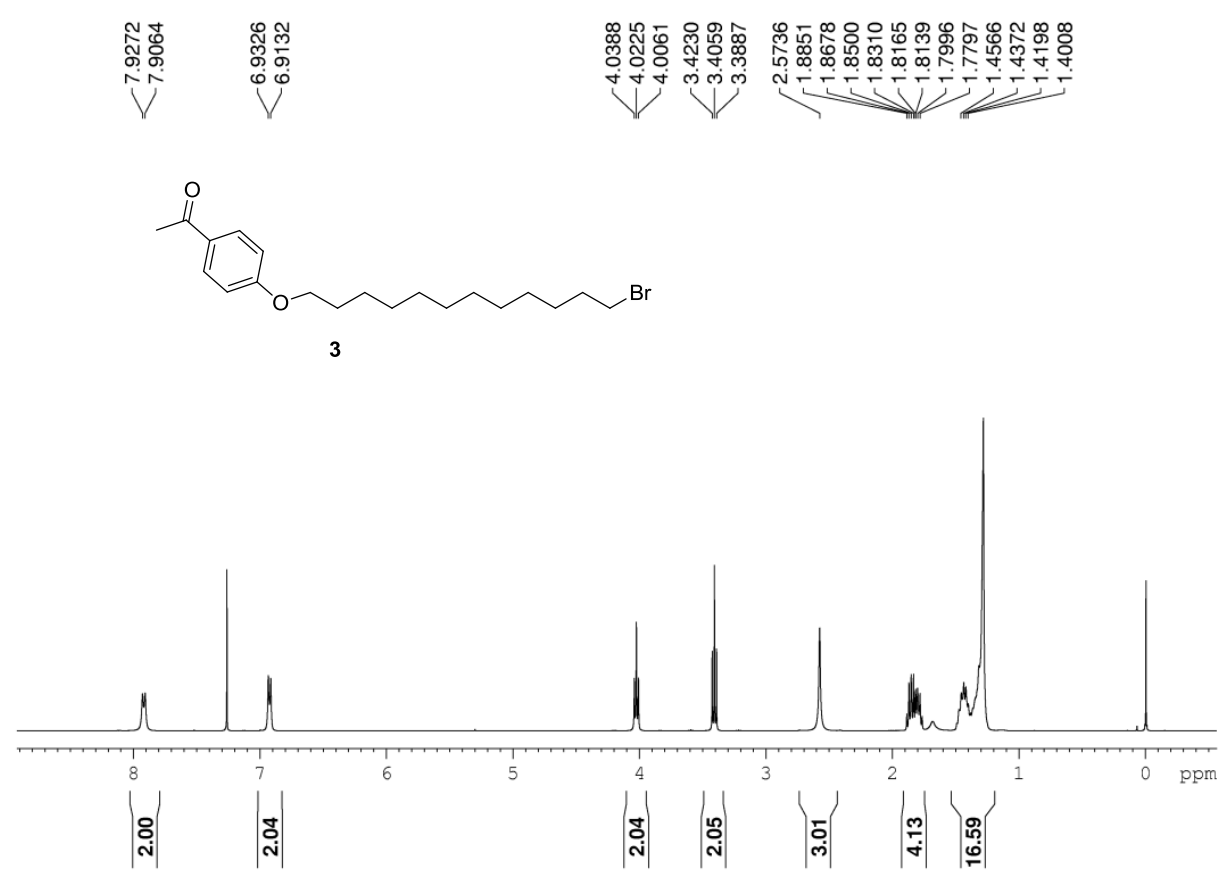

Figure S11. ${ }^{1} \mathrm{H}$ NMR Spectra of Compound 3.

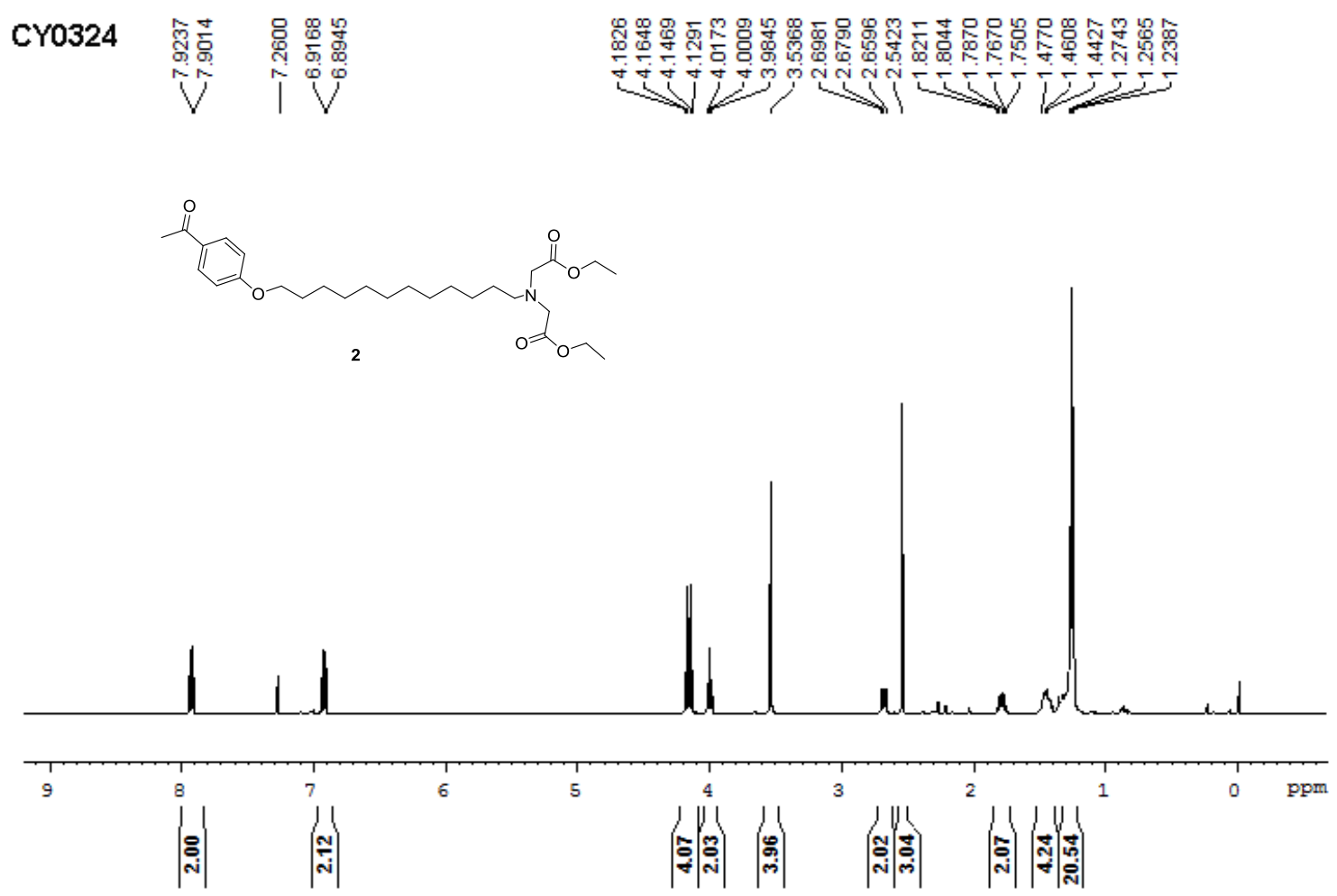

Figure S12. ${ }^{1} \mathrm{H}$ NMR Spectra of Compound 2. 


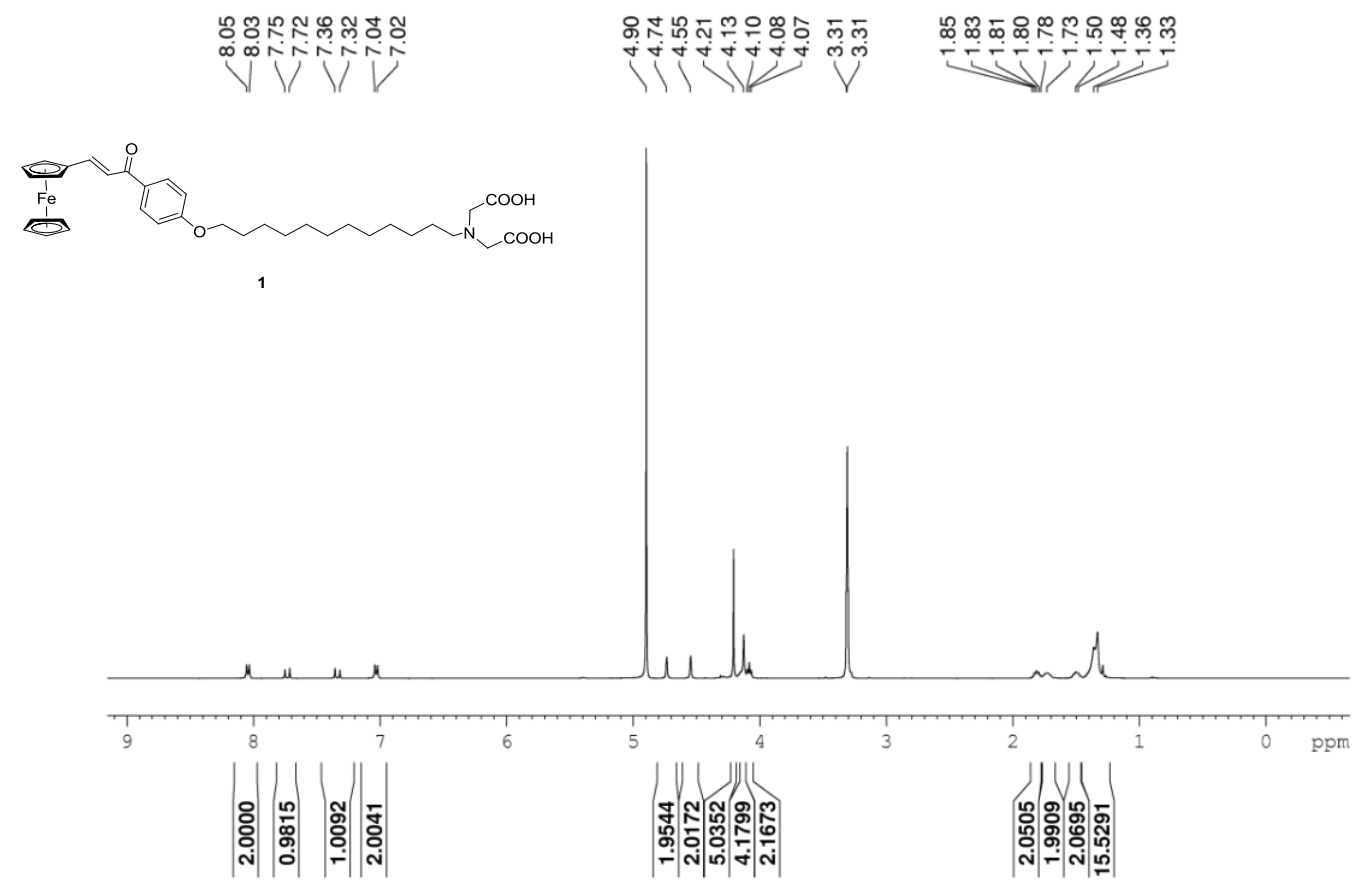

Figure S13. ${ }^{1}$ H NMR Spectra of Compound 1.

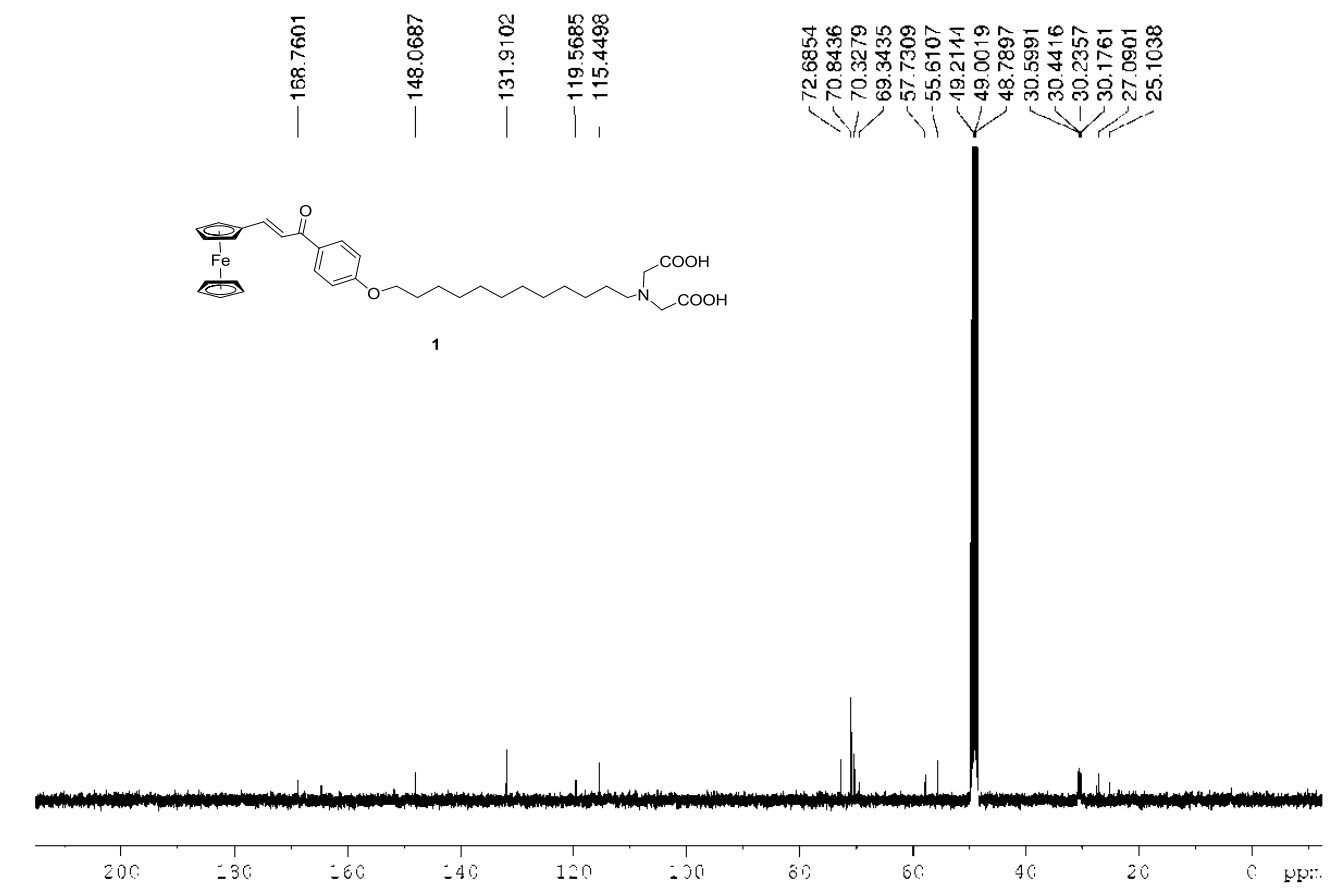

Figure S14. ${ }^{13} \mathrm{C}$ NMR Spectra of Compound 1. 


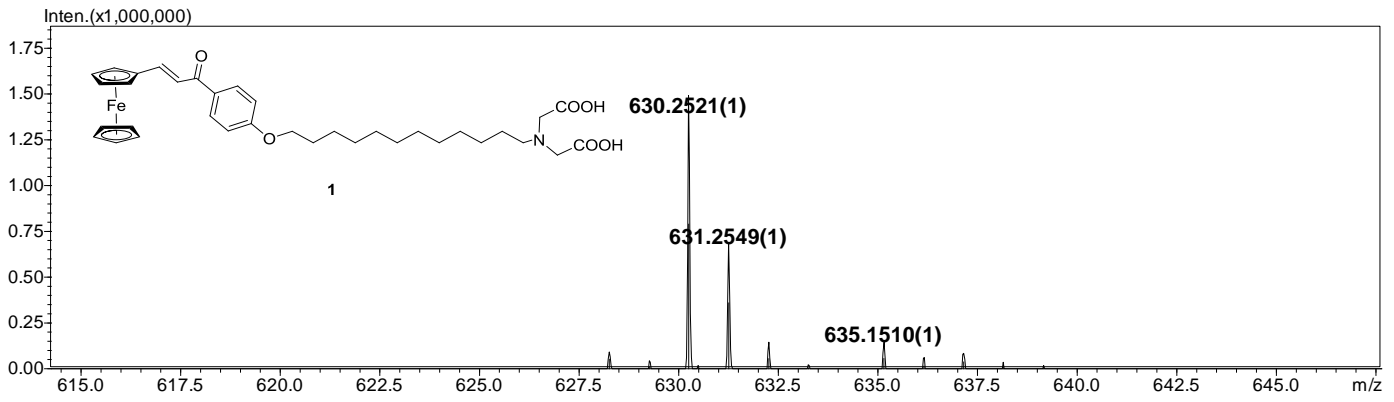

Figure S15. HR-MS Spectra of Compound 1.

Table S1. Optimization of GOx loading content in nanovesicle. The optimal loading content of GOx in nanovesicle was $47.4 \mu \mathrm{g} \mathrm{mL}^{-1}\left(8.5 \mathrm{U} \mathrm{mL}^{-1}\right)$.

\begin{tabular}{lcc}
\hline Total added GOx in solution $\left(\mu \mathrm{g} \mathrm{m}^{-1}\right)$ & Loaded GOx in vesicle $\left(\mu \mathrm{g} \mathrm{mL} \mathrm{mL}^{-1}\right)$ & GOx Loading \\
& & Content $(\mathrm{LC})(\%)^{a}$ \\
\hline 50 & 7.9 & $0.9 \% \pm 0.2$ \\
100 & 11.7 & $1.3 \% \pm 0.6$ \\
750 & 30.0 & $3.3 \% \pm 1.1$ \\
1000 & 40.4 & $4.5 \% \pm 1.5$ \\
2000 & 47.4 & $5.3 \% \pm 0.5$ \\
\hline
\end{tabular}

${ }^{a} \mathrm{GOx}$ Loading Content $\%=\left(W_{\mathrm{GOx}}\right.$ in GOx\&Pt@FcNV$\left./ W_{\mathrm{GOx} \& \mathrm{Pt} @ \mathrm{FcNV}}\right) \times 100 \%$. 
Table S2. $\mathrm{IC}_{50}$ of cisplatin, Pt@FcNV, GOx and GOx\&Pt@FcNV against MCF-7 and A549 cells at $37{ }^{\circ} \mathrm{C}$ for $24 \mathrm{~h}$.

\begin{tabular}{|c|c|c|c|c|}
\hline \multirow{2}{*}{ drug formulation } & \multicolumn{2}{|c|}{$\mathrm{IC}_{50}^{\mathrm{Pt}}\left(\mu \mathrm{g} \mathrm{mL} \mathrm{L}^{-1}\right)$} & \multicolumn{2}{|c|}{$\mathrm{IC}_{50}^{\mathrm{GOX}}\left(\mathrm{mU} \mathrm{mL} \mathrm{L}^{-1}\right)$} \\
\hline & MCF-7 & A549 & MCF-7 & A549 \\
\hline Cisplatin & 22.1 & 25.4 & - & - \\
\hline Pt@FcNV & 18.9 & 16.2 & - & - \\
\hline GOx (high glucose ${ }^{a}$ ) & - & - & 19. 4 & 25.4 \\
\hline GOx\&Pt@FcNV (low glucose $\left.\mathrm{e}^{b}\right)$ & 0.8 & 1.0 & 68.6 & 75.6 \\
\hline GOx\&Pt@FcNV (high glucose ${ }^{a}$ ) & 0.12 & 0.09 & 6.8 & 6.7 \\
\hline
\end{tabular}

${ }^{a}$ [glucose $]=4.5 \mathrm{mg} \mathrm{mL}^{-1},{ }^{b}$ [glucose $]=1.0 \mathrm{mg} \mathrm{mL}^{-1}$. 